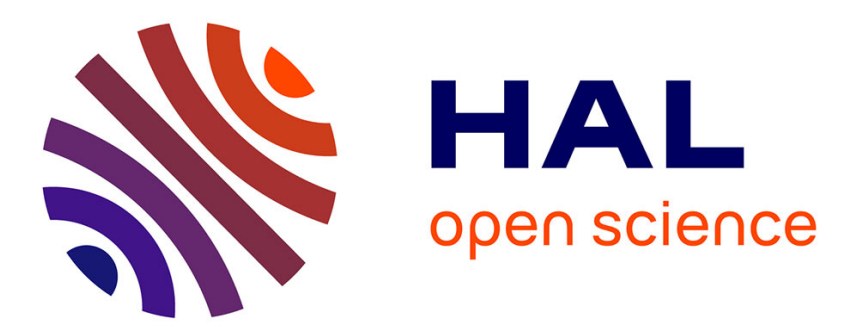

\title{
First stages of the transition to turbulence and control in the incompressible detached flow around a NACA0012 wing
}

\author{
Yannis Hoarau, Marianna Braza, Y. Ventikos, D. Faghani
}

\section{- To cite this version:}

Yannis Hoarau, Marianna Braza, Y. Ventikos, D. Faghani. First stages of the transition to turbulence and control in the incompressible detached flow around a NACA0012 wing. International Journal of Heat and Fluid Flow, 2006, 27 (5), pp.878-886. 10.1016/j.ijheatfluidflow.2006.03.026 . hal-02545290

\author{
HAL Id: hal-02545290 \\ https://hal.science/hal-02545290
}

Submitted on 16 Apr 2020

HAL is a multi-disciplinary open access archive for the deposit and dissemination of scientific research documents, whether they are published or not. The documents may come from teaching and research institutions in France or abroad, or from public or private research centers.
L'archive ouverte pluridisciplinaire HAL, est destinée au dépôt et à la diffusion de documents scientifiques de niveau recherche, publiés ou non, émanant des établissements d'enseignement et de recherche français ou étrangers, des laboratoires publics ou privés. 


\title{
First stages of the transition to turbulence and control in the incompressible detached flow around a NACA0012 wing
}

\author{
Y. Hoarau ${ }^{a, *}$ M. Braza ${ }^{a}$ Y. Ventikos ${ }^{b}$ and D. Faghani ${ }^{\mathrm{c}}$ \\ ${ }^{a}$ Institut de Mécanique des Fluides de Toulouse, France \\ ${ }^{\mathrm{b}}$ Dept of Engineering Science, University of Oxford, UK \\ ${ }^{\mathrm{c}}$ Euro-American Institute of Technology, Sophia Antipolis, France
}

\begin{abstract}
The present study analyses the successive transition steps in the flow around a high-lift wing configuration, as the Reynolds number increases in the low and moderate range $(800-10,000)$, by the Navier-Stokes approach. The flow system is mainly governed by two kinds of organised modes appearing successively as the Reynolds number increases: the von Kàrmàn and the shear layer mode. A period-doubling scenario characterises the first 2D stages of the von Kàrmàn mode up to Reynolds number 2000, where the shear-layer mode becomes predominant. The successive stages of the 3D transition are also analysed in detail. In a second step, the effect of wall suction has been studied both in 2D and 3D flows around the NACA0012 airfoil at $20^{\circ}$ of incidence and a Reynolds number of 800 . This study has the objective to optimise the aerodynamic coefficients and to attenuate the mentioned 3D transition effects in the near wake. The receptivity of the flow to the suction is clearly shown and the suction position on the wall has been optimised according to the improvement of the aerodynamics coefficients.
\end{abstract}

Key words: Instabilities, transition, drag reduction

\section{Introduction}

The transition to turbulence in the flow around airfoils has received less attention till recently, when compared to similar configurations involving bluff-body

* Corresponding author. Present adress: Institut de Mécanique des Fluides et des Solides, Strasbourg, France

Email address: hoarau@imfs.u-strasbg.fr (Y. Hoarau ). 
wakes. The main interest in the research for unsteady flows around airfoils has focused on the high-Reynolds number range and especially to pitching motion analysis related to the dynamic stall. This interest is justified by the importance of the pitching motion of lifting bodies in the Avionics industry and especially Rotorcraft industry. However, there is a major interest, both from a fundamental and industrial point of view, with particular regard to the inherent unsteady flow around airfoils and wings: the spontaneous appearance of unsteadiness with steady external conditions. There is indeed a high interest to examine the natural transition to turbulence governing this kind of flows, because of the development of main organised modes that persist at the high Reynolds number range and they interact non-linearly with any imposed (forced) frequency oscillation. From a practical point of view, the transition mechanisms lead to a substantial growth of the mean values and amplitudes of the global parameters, an issue that is important in industrial applications in aerodynamics and in fluid-structure interaction.

There are few attempts in the literature analysing the 2D unsteady separation at moderate Reynolds numbers. Mehta and Lavan (1975) in a pioneer work had simulated the starting separation vortex in a flow at low Reynolds numbers. A comprehensive review of the separation as well as of the dynamic stall can be found in McCroskey (1982). Furthermore, the birth of the natural transition in the incompressible or subsonic flow regimes were studied by Pulliam and Vastano (1993) up to the Reynolds number 3000, Ventikos et al. (1993) as well as in the transonic regime by Bouhadji and Braza (2002) up to the Reynolds number 10,000 .

Regarding the birth of the organised modes, there is little available knowledge concerning the three-dimensional mechanisms as well as the evolution of these modes over a wide, moderate Reynolds number range in the incompressible flow regime.

The present paper focuses on the early stages of the natural transition, associated with the development of organised modes as the Reynolds number increases. The geometry chosen is the NACA0012 wing at a high angle of incidence $20^{\circ}$, ensuring a massively detached flow in a wide Reynolds number range. This study is based on time-dependent Navier-Stokes simulation, in two and three dimensions. The following points will be discussed:

- Identifying the successive changes that the flow undergoes as the Reynolds number increases, as well as the related instability modes.

- Analysing in detail the shear-layer transition under the non-linear interaction with the von Kàrmàn mode in the higher Reynolds number range.

- Analysing the development of the 3D transition from a nominally $2 \mathrm{D}$ flow configuration subjected the von Kàrmàn mode, that is strongly asymmetric, due to the lifting body configuration. 
- Decomposition and reconstruction of the flow with the Proper Orthogonal Decomposition method

The second part of this study deals with the effect of wall suction on a massively detached flow around a NACA0012 airfoil at $20^{\circ}$ of incidence and a Reynolds number of 800. 2D laminar computations have been performed as well as 3D computations where the effects of the suction on the 3D structures are presented.

\section{Governing equations and numerical method}

The governing equations are the continuity and the Navier-Stokes equations for an incompressible fluid. The numerical method is based on the pressurevelocity formulation employing a predictor-corrector pressure scheme and the staggered grids of velocity and pressure. Two algorithms of similar features, both based on this methodology have been used for the 2D study, both leading to practically the same results. The first one is an implicit form of an originally explicit scheme by Amsden and Harlow (1970), extended in the present case to an implicit scheme for unsteady flows, by Braza et al. (1986). The Navier-Stokes equations are solved by using a general curvilinear conformal mapping, Thomson et al. (1974)). The time discretisation in 3D is achieved by an Alternating Direction Implicit fractional-step scheme, originally studied by Douglas (1962) for diffusion type equations and extended in the present study in the whole convection-diffusion Navier-Stokes systemfor unsteady flows. This scheme employes an Alternating Direction Implicit method, leading to the solution of tri-diagonal systems by a very fast algorithm. The time accuracy is of second-order. The space discretisation is done by using central differences providing a second-order of accuracy. The wall pressure is discretised by the exact form of the Navier-Stokes equations at the wall, and not by using approximate Neumann-type conditions as usually. This ensures an accurate evaluation of the wall pressure especially in the strongly detached flow regions in 3D. These overall aspects constitute the EMT2/IMFT code ICARE, widely used in an academic research context. The details of the numerical scheme in 3D can be found in Persillon and Braza (1998). The code ICARE is fully parallelised in MPI architectures, (Hoarau et al. (2001)) and allows the use of a high number of parallel processors and therefore, significant grid sizes for the DNS approach. This is decisive for realistic 3D computations. The second algorithm used is a SIMPLE-type fully implicit algorithm, whose description can be found in Tzabiras et al. (1986). The discretisation of the space derivatives is done by using central differences. Orthogonal curvilinear staggered grids are employed. The methodology is second-order accurate in space and time. The equations are written in orthogonal curvilinear systems. 


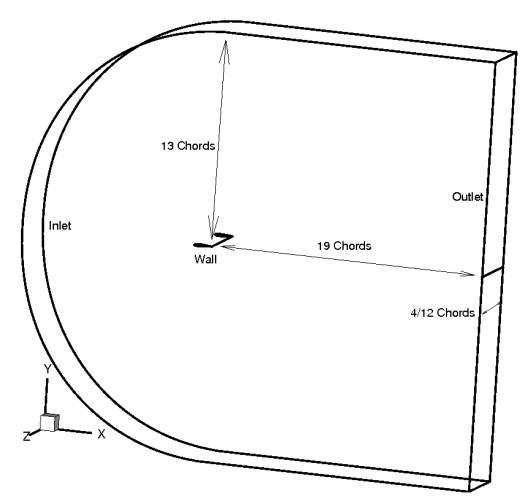

a)

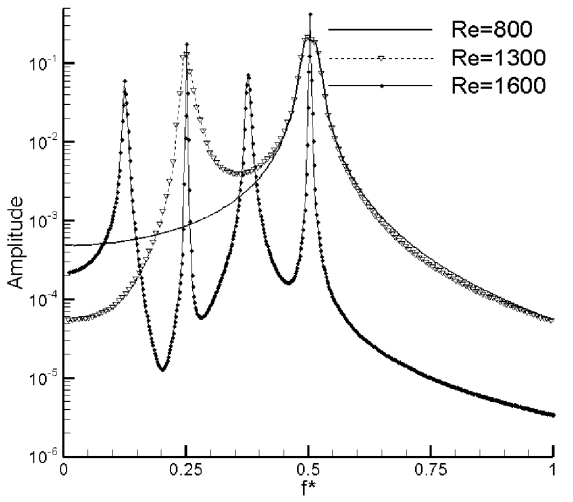

b)

Fig. 1. Computational domain and Pressure coefficient spectra showing the period doubling mechanism

Figure 1a shows an overview of the computational domain. The boundary conditions are free-stream at the inlet boundary and non-reflective absorption conditions for the outlet boundary, specified by Jin and Braza (1993) to minimise very efficiently any feedback effect in the incompressible flow regime. The impermeability and adherence conditions are specified for the solid wall. C type grids are employed in both cases: typical grids size used for the $2 \mathrm{D}$ simulations are $250 \times 160,450 \times 200$ to $1000 \times 400$ and the time-step is $\Delta t=0.001$. The grid used for the $3 \mathrm{D}$ simulations is $(413 \times 70 \times 90)$. The spanwise length of the computational domain is $4 c$ and $12 c$, both providing the same kind of three-dimensional phenomena. $c$ represents the chord length and $\operatorname{Re}=U_{\infty} c / \nu$ is the Reynolds number.

A very detailed and careful study of the numerical parameters and of the dimensions of the computational domain had been conducted for the final choice, in respect of the grid and the spanwise distance independence of the results, as well as for the 2D study (Hoarau (2002), Ventikos (1995)).

The computations are carried out on the supercomputers SP3, SP4 and Origin 3000 of the national supercomputer centers CINES, IDRIS and CALMIP.

\section{Instability and transition to turbulence}

\subsection{The first steps of $2 D$ transition}

Firstly, computations are carried out at the very low Reynolds number regime (around $\mathrm{Re}=5$ ) showing a fully attached steady flow. As the Reynolds number increases, a small attached vortex is created near the trailing edge and grows with the Reynolds number. Near Reynolds number 70, where the bubble has 


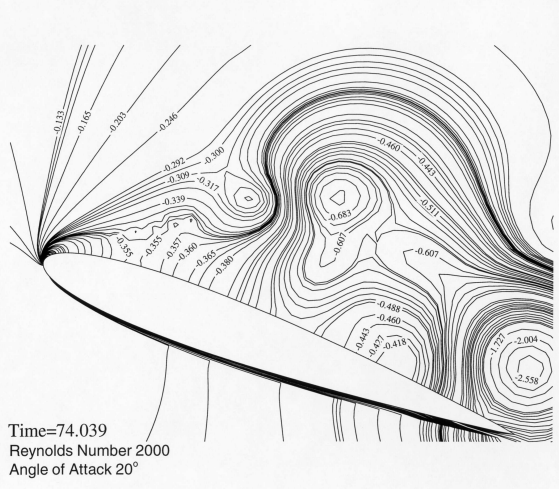

a) $\mathrm{Re}=2000$

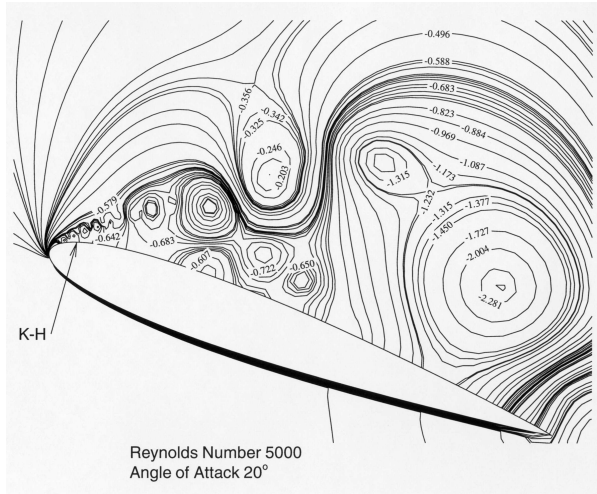

d) $\quad \operatorname{Re}=5000$

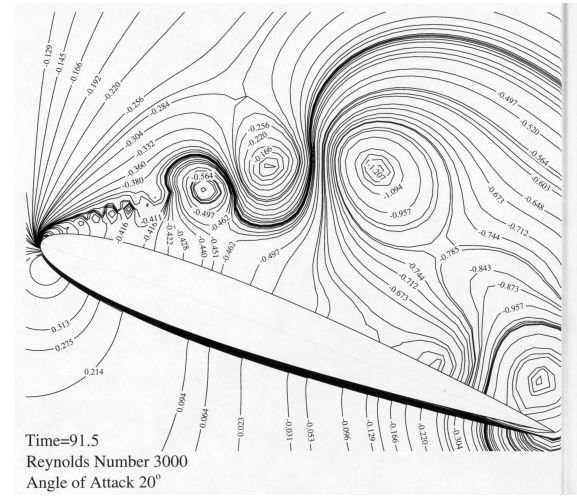

b) $\mathrm{Re}=3000$

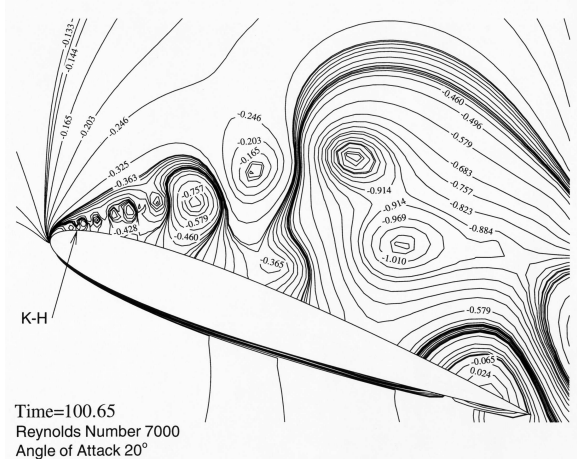

e) $\quad \operatorname{Re}=7000$

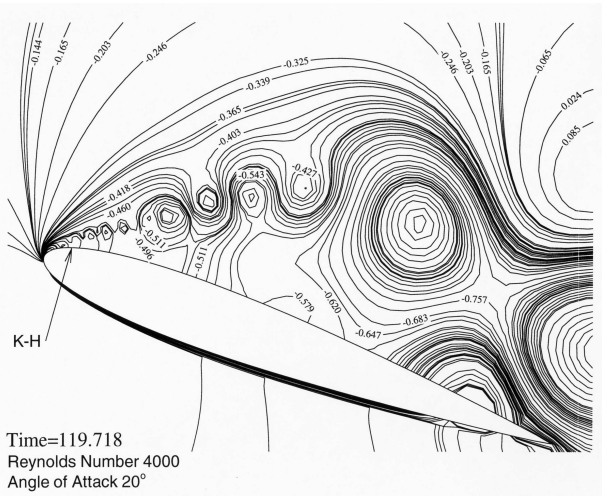

c) $\operatorname{Re}=4000$

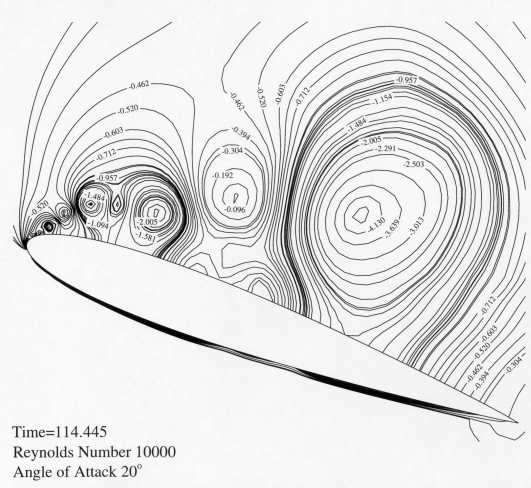

f) $\mathrm{Re}=10000$

Fig. 2. Instantaneous iso-pressure contours: spatial view of the shear-layer instability as Reynolds number increases

attained a nearly $40 \%$ length relatively to the chord, unsteady separation starts and a very regular vortex shedding appears. The vortex shedding pattern is attained at $R e=450$. At $R e=800$, the vortex shedding motion is very regular. This regime is analysed in detail in the present study with respect to the $2 \mathrm{D}$ and 3D transition mechanisms. Beyond the Reynolds number 800, the vortex shedding regularity is attenuated and other predominant frequencies appear, being fractions of the fundamental, up to $R e=2000$. As the Reynolds number increases further on, the transition process becomes more complex, because of the development of a shear-layer instability as an incommensurate mode. In fact, two different mechanisms can be identified as the Reynolds number increases: the period-doubling mechanism and the shear-layer instability.

The first mechanism corresponds to the evolution of the von Kàrmàn instability and it is closely related to a period doubling scenario as is clearly shown in the spectra of the numerically obtained signals for increasing values of the Reynolds number in the range (800-1600), (fig. 1b). The appearance of the first subharmonic frequency of the main vortex shedding one can be physically justified by the fact that at a higher Reynolds number value, the shed vortex close 
to the trailing edge is weakened at exactly $2 \mathrm{~T}$ ( $\mathrm{T}$ being the vortex shedding period) by the opposite vortex which starts to be shed. This illustrates the phenomenon of period doubling, that is characterised by an energy - vorticity exchange process. This mechanism becomes predominant whenever the externally supplied energy (Reynolds number) to the system becomes higher than a critical value, in the context of the non-antisymmetric vortex shedding mode, owing to the lifting-body configuration. It will be remembered that this kind of mechanism does not appear in the case of a symmetric von Kàrmàn vortex shedding, as for example in flows past bluff bodies. The period doubling mechanism appears repetitively as the Reynolds number increases further on, and it yields spectra with four, eight, ... peaks. Our results compare very favourably with the ones obtained by Pulliam and Vastano (1993). These authors have shown that the period doubling continues further on, as the Reynolds number increases.

The second mechanism appears beyond $R e=2,000$. The separated shear layer undergoes another important transition mechanism that gives rise to an incommensurate frequency, due to the development of a Kelvin-Helmholtz instability. In the present case, this instability mode is forced by the oscillatory motion of the separation point, that obeys the von Kàrmàn instability. Figure 2 show the clear formation of Kelvin Helmholtz vortices. The length of the shear-layer vortices is smaller than the von Kàrmàn ones and it decreases as the Reynolds number increases. A detailed space-time tracking of these vortices allows the evaluation of the shear-layer instability wavelength in the present case. It is found that the wavelength decreases as the Reynolds number increases, according to the law $\lambda_{s l} \propto \mathrm{Re}^{-0.44}$. Furthermore the variation law of ratio $\mathrm{f}_{s l} / \mathrm{f}_{V-K}$ is $\propto \mathrm{Re}^{0.43}$. This law exponent is very close to $\mathrm{Re}^{0.5}$ that characterises the development of the instability wave prior to separation, as reported by Bloor (1964) concerning bluff-body wakes.

The shear-layer frequency is an incommensurate mode in comparison to the von Kàrmàn mode. This leads, in association with the period-doubling scenario, to the non-linear filling-up of the energy spectrum by a multitude of modes, that are combinations of the von Kàrmàn and of the shear-layer mode, in the same way as reported by Braza et al. (1990) for bluff-body wakes. The variation of the global parameters versus the Reynolds number is shown in figure 3a. The mean lift coefficient undergoes a smooth decrease as the Reynolds number increases, because the flow is already stalled at $20^{\circ}$, for all the Reynolds numbers examined. Therefore, this decrease corresponds to an equivalent behaviour occurring at fixed Re and increased incidence beyond stall. For the same reasons the drag coefficient shows a plateau saturation level, attained in the intermediate Reynolds number range, to decrease further on as a function of the Reynolds number. The more abrupt decrease of the drag coefficient beyond the Reynolds number 5,000 is a consequence of the multitude of the shear-layer vortices and of the formation of a quasi-stagnation 


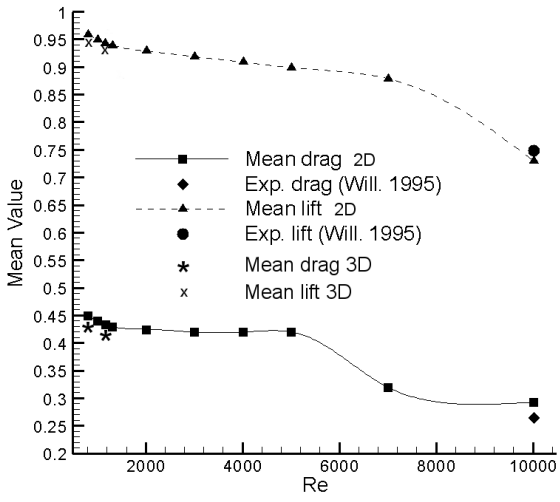

a)

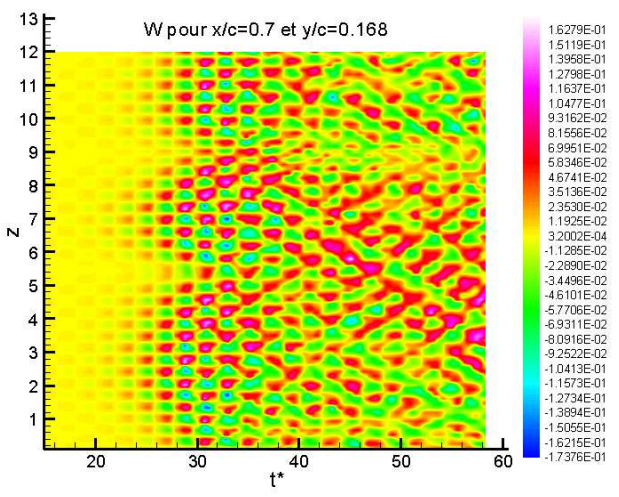

b)

Fig. 3. Evolution of the global parameters and of the spanwise velocity structure; (a) mean drag and lift coefficients versus Re and (b) time-space evolution of the $w$ velocity component along the span at $\mathrm{Re}=800$

region below the separated shear layer, up to the wall.

The Strouhal number evaluated from the lift coefficient directly obeys the shedding motion of the lower trailing edge vortex, that is delayed by the creation of the above-mentioned fully developed region beyond $\mathrm{Re}=5000$. Therefore, this step is qualitatively similar to the 'drag crisis' appearing in bluffbody wakes at higher Reynolds number, although the wake formation region of a circular cylinder varies significantly in this range. In the critical regime, a multitude of small-scale vortices are created upstream of the separation, because of the boundary-layer transition occurring upstream of the separation. The Strouhal number also shows a decrease as the Reynolds number increases due to the same reasons. These effects are obtained in the present study by the completely non-linear approach of the Navier-Stokes system. A comparison of the above global parameters is done with a water-channel experiment Williamson et al. (1995), where pressure and forces measurements were carried out by means of pressure transducers and forces balance, as well as pressure fluctuation spectra. A good agreement is obtained at Reynolds number 10,000 between the present simulation and the experiment. Furthermore, the 3D computations in the low Re-range found that the Strouhal number is essentially the same as in the 2D case. These facts ensure the validity of the numerical study from the low to the higher Reynolds number range.

\subsection{The first steps of $3 D$ transition}

In this section the way of the development of the $3 \mathrm{D}$ transition to turbulence from a nominally 2D flow configuration is examined. The Reynolds number 800 has been selected first, because it corresponds to a very strong and regular development of the von Kàrmàn mode. The initial conditions are either 
those of a flow at rest triggered by a very weak spanwise $w$ velocity fluctuation imposed as a random fluctuation, or a fully developed $2 \mathrm{D}$ vortex shedding pattern perturbed in the same way. The dimensionless $r m s$ values of the spanwise fluctuation are of order $10^{-4} U_{\infty}$. This technique does not privilege the appearance of any wavelength and the order of magnitude of the fluctuation is very weak and less than the physically existing upstream noise in any wind tunnel. By performing a very detailed 3D study, it has been found that the flow 'forgets' its initial conditions and both ways of initiating the 3D transition lead to the same final regime: the first step is the development of the $2 \mathrm{D}$ von Kàrmàn pattern followed by the appearance of the $3 \mathrm{D}$ mechanisms as described below.

Figure $3 \mathrm{~b}$ shows the time-space evolution of the $w$ velocity component along the span. After a transient phase, the onset of the 3D transition appears as the organised pattern of the iso- $w$ velocity contours according to coherent counter-rotating cells. This step is followed by the amplification of the longitudinal and vertical vorticity components (fig. 4a-b), that are found to form the same kind of coherent cells. This spanwise-periodic fluctuation plays the role of a perturbing factor acting on the von Kàrmàn rectilinear vortex rows. Consequently, the $\omega_{z}$ vorticity is modified according to the vorticity conservation equations (fig 4c). Following the elliptic stability theory (Landman and Saffman (1987)), the expected spanwise mode of an originally 2D elliptic-shape vortex (in the present case the von Kàrmàn vortex rows) is a 3D-undulated large-scale vortex row according to a regular spanwise wavelength.

The dynamics of this pattern are similar to the ones of bluff-body wakes DNS studies, (Persillon and Braza (1998)), but in the present case the shearing mechanism is totally asymmetric. The shape of the undulated vortices is much more stretched, according to the lifting body configuration. By performing a space-averaging of all the 3D transverse sections at the same instant, it has been proven that the alternating vortex pattern is very similar to the corresponding $2 \mathrm{D}$ configuration at the same phase. Therefore, the present 3D route to transition is expected to be affected by the same kind of perioddoubling cascade and of the shear-layer instability, as discussed in the 2D study. However, the present 3D study is still carried out in a low Reynolds number range where these effects are not yet fully pronounced. By carrying out a Fast Fourier Transform analysis of the spanwise evolution of the secondary instability mode, it has been feasible to evaluate the preferential spanwise wavelength developed under the present conditions, $\lambda_{z} / D=0.64$. This value is found in good agreement with the results concerning bluff body wakes: although the fundamentals of the shearing mechanism are different in the present case of lifting body wakes, an analogy with the bluff body ones can be done by considering an 'equivalent' bluff body configuration having a characteristic vertical distance $c^{*} \sin (\alpha)$ in respect of the upstream velocity direction, $c$ being the chord and $\alpha$ the incidence. Therefore, the "effective" Reynolds 


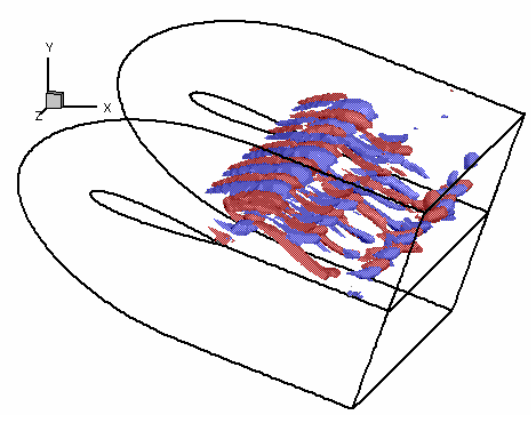

a) $\omega_{x}= \pm 2$

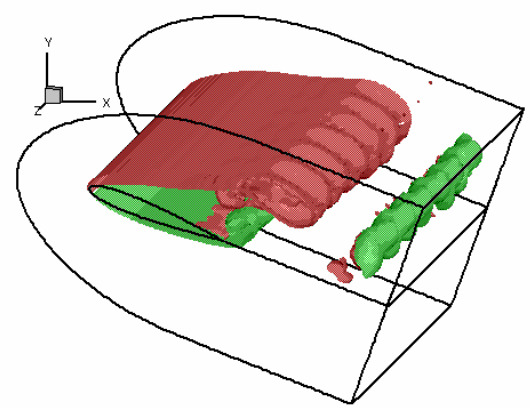

c) $\omega_{z}=4.1$

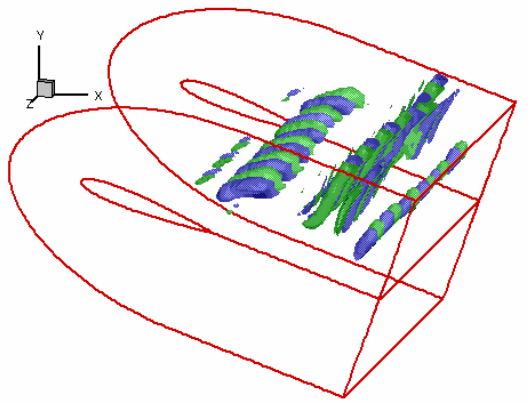

b) $\omega_{y}= \pm 2$

Fig. 4. Spanwise evolution of the vorticity

number in the analogy bluff-body and wing-body is $R e{ }^{*} c{ }^{*} \sin \left(20^{\circ}\right)=273$. The expected wavelength for this 'equivalent' bluff-body wake would be of order 0.60-0.70 according to the DNS by Persillon and Braza (1998), Braza et al. (2001). The same kind of 3D dynamics govern the flow at $\mathrm{Re}=1200$ and the corresponding wavelength $\lambda_{z} / \mathrm{D}=0.62$, at an effective $\mathrm{Re}=410.5$.

The secondary instability development is expected to continue as Re increased by providing progressively smaller wavelengths, together with the development of the previous mentioned cascades. Because of the robustness of the $2 \mathrm{D}$ alternating pattern even at higher Re, it is expected that both routes to transition persist and be clearly identified as Re further increases, by involving in addition non-linear effects. This study is an immediate outlook of the present one, by our research group.

\subsection{Low-order decomposition and reconstruction}

The Proper orthogonal decomposition analysis of the flow system in the sense of a "Low Order Dimensionning", L.O.D. system has been performed by means 
of the DNS data, stored with a considerable detail in space and time. From a data set $\overrightarrow{u_{i}}(\vec{x}, t)$ the Proper Orthogonal Decomposition analysis consists in searching the function that is most similar to the members of $\overrightarrow{u_{i}}(\vec{x}, t)$ on average (Berkooz et al. (1993)). This is done by solving an eigenvalue problem where the kernel is the two point correlation tensor .

Because of the detailed representation of the results basis offered by the DNS, the B.O.D. (bi-orthogonal decomposition) has been applied in the present complete data set, according to Aubry et al. (1991) instead of the snapshots method usually applied in experimental data (Barthet (2003), Faghani (1993)). For this method we split the field $\overrightarrow{u_{i}}(\vec{x}, t)$ in time and space as:

$$
\vec{u}_{i}(\vec{x}, t)=\sum_{n=1}^{\infty} a_{n} \psi_{n}(t) \vec{\phi}_{i, n}(\vec{x})
$$

where $\phi(\vec{x})$ is the spatial part, called topos and $\psi(t)$ is the temporal part called chronos. The eigenvalues ares computed for the two kernels:

$$
\begin{aligned}
& l_{i j}\left(\vec{x}, \vec{x}^{\prime}\right)=\int u_{i}(\vec{x}, t) u_{j}\left(\vec{x}^{\prime}, t\right) d t \\
& s\left(t, t^{\prime}\right)=\int u_{i}(\vec{x}, t) u_{i}\left(\vec{x}, t^{\prime}\right) d \vec{x}
\end{aligned}
$$

The equations associated to the eigenvalues of these kernels can be written as:

$$
\begin{aligned}
& \int l_{i j}\left(\vec{x}, \vec{x}^{\prime}\right) \phi_{i, n}\left(\vec{x}^{\prime}\right) d \vec{x}^{\prime}=a_{n}^{2} \phi_{i, n}(\vec{x}) \\
& \int s\left(t, t^{\prime}\right) \psi_{n}\left(t^{\prime}\right) d t^{\prime}=a_{n}^{2} \psi_{n}(t)
\end{aligned}
$$

We applied this decomposition to the flow around a NACA0012 at a Reynolds number of 1200 and $20^{\circ}$ of incidence. More than 6000 3D snapshots were used to compute the bi-orthogonal decomposition. Figures 5 show the B.O.D. reconstruction of the flow system and the energy drop of the P.O.D. modes. It is shown that the first five modes are the most energetic and are sufficient to reproduce the von Kàrmàn instability but at least a 20-mode reconstruction is needed to describe both the coherent structures and the secondary instability of the present system.

\section{Control of instabilities}

In this part we study the effect of a constant suction at the wall on the aerodynamics coefficients and on the second instability of a NACA0012 airfoil 


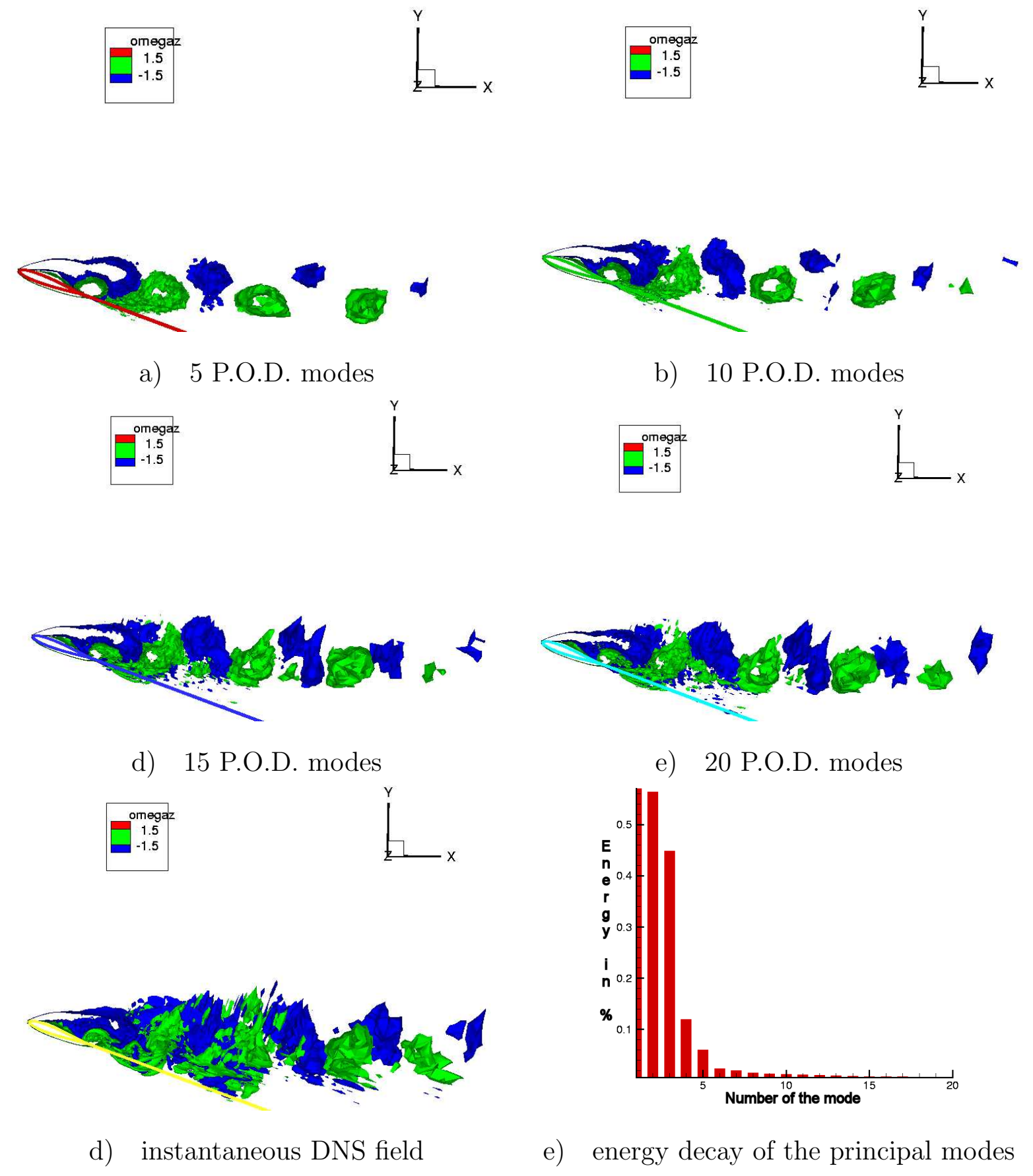

Fig. 5. Iso-vorticity lines of the P.O.D. reconstructions of the 3D secondary instability pattern

at $20^{\circ}$ of incidence and for a Reynolds number of 800 . We first studied the effect of the position of the suction on the aerodynamic coefficients and then used the best position to study 3D effects. 


\begin{tabular}{|l|c|c|}
\hline & Drag Coefficient $\mathrm{C}_{x}$ & Lift coefficient $\mathrm{C}_{z}$ \\
\hline Without suction & 0.435 & 0.968 \\
\hline $\mathrm{x} / \mathrm{c}=0.016, \mathrm{D}=9.0410^{-3}$ & 0.361 & 0.899 \\
\hline $\mathrm{x} / \mathrm{c}=0.063, \mathrm{D}=1.0310^{-2}$ & 0.34 & 0.96 \\
\hline $\mathrm{x} / \mathrm{c}=0.063, \mathrm{D}=2.0710^{-2}$ & 0.285 & 1.071 \\
\hline $\mathrm{x} / \mathrm{c}=0.114, \mathrm{D}=1.0310^{-2}$ & 0.389 & 0.972 \\
\hline $\mathrm{x} / \mathrm{c}=0.166, \mathrm{D}=1.0410^{-2}$ & 0.418 & 0.961 \\
\hline $\mathrm{x} / \mathrm{c}=0.218, \mathrm{D}=1.0410^{-2}$ & 0.428 & 0.95 \\
\hline $\mathrm{x} / \mathrm{c}=0.270, \mathrm{D}=1.0410^{-2}$ & 0.432 & 0.941 \\
\hline $\mathrm{x} / \mathrm{c}=0.322, \mathrm{D}=1.0410^{-2}$ & 0.433 & 0.961 \\
\hline $\mathrm{x} / \mathrm{c}=0.374, \mathrm{D}=1.0310^{-2}$ & 0.436 & 0.93 \\
\hline $\mathrm{x} / \mathrm{c}=0.439, \mathrm{D}=1.3110^{-2}$ & 0.439 & 0.928 \\
\hline
\end{tabular}

Table 1

Evolution of the aerodynamics coefficients with the position of the suction.

\subsection{Two-dimensional case}

The suction is applied to an initial field already submitted to the von Kàrmàn instability. The suction is a generated by modifying the non-slip condition at the wall and using a negative vertical velocity. Many positions have been tested and the results on the aerodynamics coefficients are shown on the table 4.1 , where $\mathrm{D}$ is the normalised debit $D=V_{\text {suction }} * l_{\text {suction }}$.

The best position is found to be at $x / c=0.063$ for a suction vertical velocity $v=-0.4$. This position corresponds to the beginning of the detachment on the extrados. It is noticeable that the suction doesn't kill the von Kàrmàn instability although it has favourable effect of the drag and lift coefficients.

\subsection{Three-dimensional case}

In this last part we have chosen the optimum position found in the $2 \mathrm{D}$ study and we performed 3D (DNS) computations. The initial flow field is one already submitted to the first instability (von Kàrmàn) and the second (spanwise undulation). The suction has been applied in the different ways: every $\lambda_{z}$ in the spanwise direction $\left(\lambda_{z}\right.$ is the spanwise wavelength of the secondary instability), every $\lambda_{z} / 2$ and continuously.

Figure 6 shows that each configuration has a positive impact on the coeffi- 


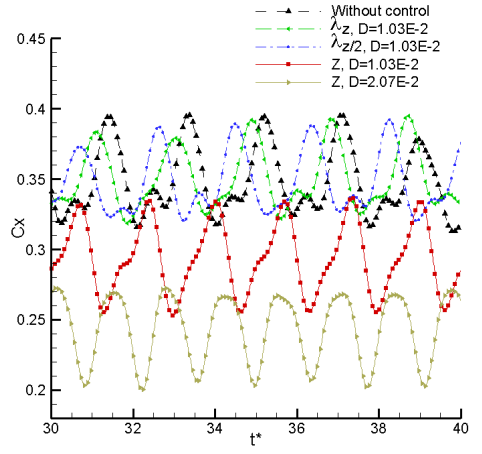

a) Drag

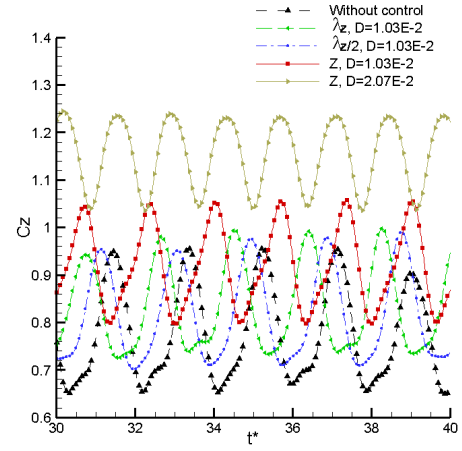

b) Lift

Fig. 6. Effect of the suction on the 3D aerodynamic coefficients

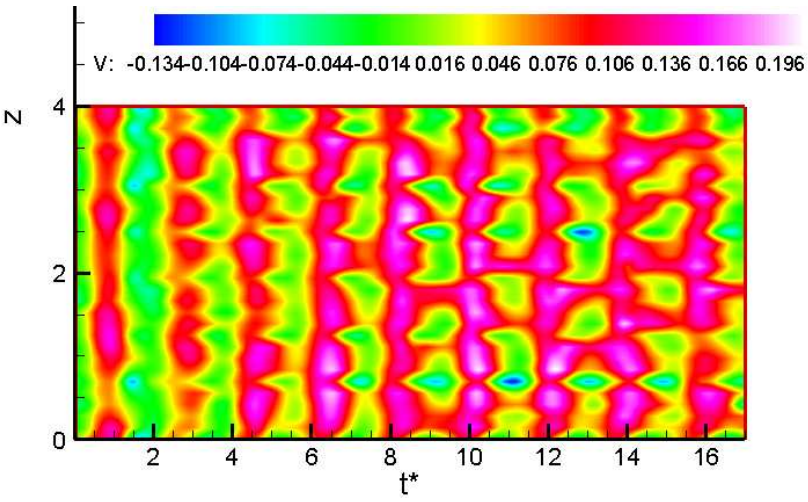

a)

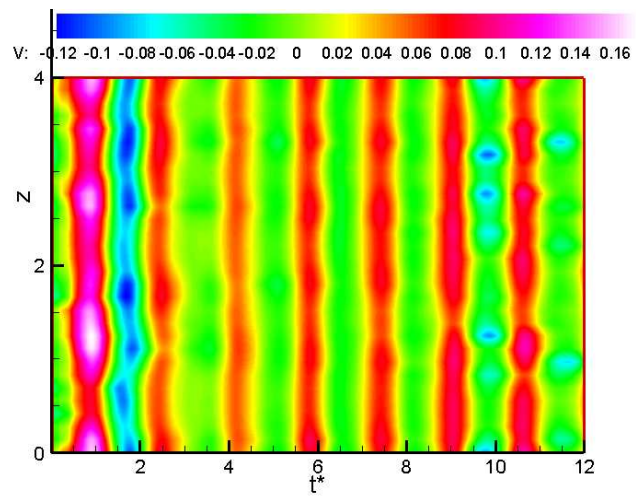

b)

Fig. 7. Time and space evolution of the vertical velocity in the recirculation area for a suction applied every $\lambda_{z}$ (a) and continuously (b)

cients but the suction applied continuously in the spanwise direction give the best results. The suction speed of -0.4 provides better results than the -0.2 one. In fact the suction applied periodically along the span according to the natural wavelength distance, acts as a forcing that improves the aerodynamic coefficients but has little effect on the three-dimensionality of the flow in the near wall region. The suction applied continuously along the span indeed attenuates the spanwise undulation (fig. 7). In the case of a continuous suction, the flow becomes practically $2 \mathrm{D}$ in the near wall region and weakly $3 \mathrm{D}$ in the far region.

\section{Conclusion}

The present study analyses the successive transition steps in the flow around a high-lift wing configuration, as the Reynolds number increases in the low and 
moderate range (800-10,000), by the Navier-Stokes approach. A quite good comparison is performed with a water-channel experiment.

According to a $2 \mathrm{D}$ study, it is found that the present flow system is mainly governed by two kinds of organised modes appearing successively as the Reynolds number increases, the von Kàrmàn and the shear layer mode. A perioddoubling scenario characterises the first 2D stages of the von Kàrmàn mode.

The analysis of the shear-layer mode in the flow around an airfoil as a function of the Reynolds number is carried out. The variation law of the predominant streamwise wavelength and of the shear-layer frequency are determined versus the Reynolds number.

The successive stages of the 3D transition around a lifting body beyond the first bifurcation are analysed in detail in the low Reynolds number range (8001200). The history of the three-dimensional modes development, the robustness of the alternating vortex pattern and the quantification of the spanwise predominant wavelengths are clearly shown.

The effect of a wall suction around a NACA0012 airfoil at $20^{\circ}$ of incidence and a Reynolds number of 800 has been studied. The optimal position for the suction has found at the beginning area of the detachment. Improvements of the aerodynamic coefficients in the 2D case are achieved, confirmed in the 3D case. Furthermore, an attenuation on the secondary instability has been achieved by employing the suction technique along the span.

\section{References}

Amsden, M. A., Harlow, F. H., 1970. The SMAC method : a numerical technique for calculating incompressible fluid flows. Los Alamos Scientific Laboratory Report. L.A. 4370.

Aubry, N., Guyonnet, R., Lima, R., 1991. Spatio-temporal analysis of complex signals : Theory and applications. J. of Statistical Physics 64.

Barthet, A., 2003. Analyse structurelle des propriétés de la turbulence 3d instationnaire autour d'une aile par simulation numérique directe. DEA INPT, 25 June 2003.

Berkooz, G., Holmes, P., Lumley, J. L., 1993. The proper orhogonal decomposition in the analysis of turbulent flows. Ann. Rev. Fluid Mech. 25, 539-575.

Bloor, M., 1964. Transition to turbulence in the wake of a circular cylinder. J. Fluid Mech. 19, 290-304.

Bouhadji, A., Braza, M., 2002. Organised modes and shock-vortex interaction in unsteady transonic flows around an aerofoil. Part I and II. J. Computers and Fluids In print.

Braza, M., Chassaing, P., Ha-Minh, H., 1986. Numerical study and physical 
analysis of the pressure and velocity fields in the near wake of a circular cylinder. J. Fluid Mech. 165, 79-130.

Braza, M., Chassaing, P., Ha-Minh, H., 1990. Prediction of large-scale transition features in the wake of a circular cylinder. Phys. Fluids A2, 1461-1471.

Braza, M., Persillon, H., Faghani, D., 2001. Successive stages and the role of natural vortex dislocations in three-dimensional wake transition. J. Fluids Mech. 439, 1-41.

Douglas, J., 1962. Alternating direction methods for three space variables. Numerische Mathematik 4, 41-63.

Faghani, D., 1993. étude des structures tourbillonaires de la zone proche d'un jet plan: approche non stationnaire multidimensionnelle. Thèse de Doctorat de l'INPT.

Hoarau, Y., 2002. Analyse physique par simulation numérique et modélisation des écoulements décollés instationnaires autour de surfaces portantes. Thèse de Doctorat de l'INPT.

Hoarau, Y., Rodes, P., Braza, M., Mango, A., Urbach, G., Falandry, P., Battle, M., 2001. DNS of the 3D transition to turbulence in the incompressible flow around a wing by a parallel implicit navier-stokes solver. In: Proceeding of the Parallel CFD 2000,Trondheim. Elsevier Science B. V., pp. 433-440.

Jin, G., Braza, M., 1993. A non-reflecting outlet boundary condition for incompressible unsteady Navier-Stokes calculations. J. Computational Physics 107, 239.

Landman, M., Saffman, P., 1987. The three-dimensionnal instability of strained vortices in viscous fluid. Phys. Fluids, 2339-2342.

McCroskey, W. J., 1982. Unsteady airfoils. Ann. Rev. Fluid Mech. 14, 285-311.

Mehta, U. B., Lavan, Z., 1975. Starting vortex, separation bubbles and stall: A numerical study of laminar unsteady flow around an airfoil. J. Fluid Mech. 67, 227-256.

Persillon, H., Braza, M., 1998. Physical analysis of the transition to turbulence in the wake of a circular cylinder by three-dimensional navier-stokes simulation. J. Fluid Mech. 365, 23-88.

Pulliam, T., Vastano, J., 1993. Transition to chaos in an open unforced 2d flow. J. Computational Physics 105.

Thomson, J., Thames, F., Martin, C., 1974. Automatic numerical generation of body-fitted curvilinear coordinate system for flows containing any number of arbitrary two-dimensional bodies. J. Computational Physics 15, 299-319.

Tzabiras, G., Dimas, A., Loukakis, T., 1986. A numerical method for the calculation of incompressible, steady, separated flows around aerofoils. Int. J. Num. Meth. in Fluids 6(11), 789-809.

Ventikos, Y., 1995. Numerical investigation of unsteady, cavitating and noncavitating flows around hydrofoils. PhD Thesis, National Technical University of Athens.

Ventikos, Y., Tzabiras, G., Braza, M., 1993. The effect of viscous dissipation on the organised structures in the wake past an aerofoil in transition to turbulence. In: Ninth Symposium on "Turbulent Shear Flows", Kyoto, Japan, 
August 16-18.

Williamson, C., Govardhan, R., Prasad, A., 1995. Experiments on low Reynolds number NACA0012 aerofoils. Tech. rep., Cornell University. 